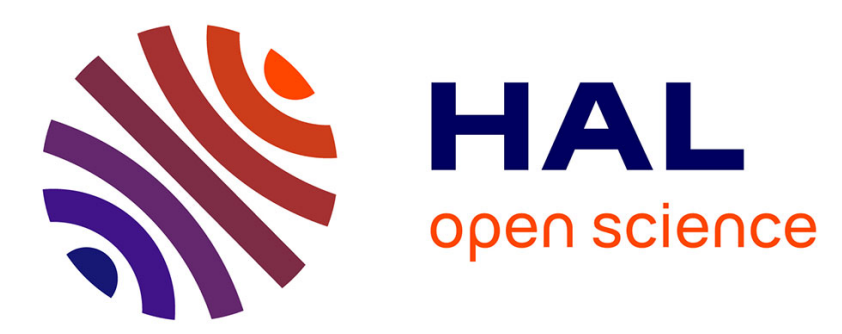

\title{
Ultrathin junctions based on the LaSrMnO_3/Nb:SrTiO_3 functional oxide interface
}

G. Kurij, L.E. Calvet, R. Guerrero, T. Maroutian, G. Agnus, A. Solignac, Ph. Lecoeur

\section{- To cite this version:}

G. Kurij, L.E. Calvet, R. Guerrero, T. Maroutian, G. Agnus, et al.. Ultrathin junctions based on the LaSrMnO_3/Nb:SrTiO_3 functional oxide interface. Thin Solid Films, 2016, 617, pp.82 - 85. 10.1016/j.tsf.2016.03.062 . cea-01485765

\section{HAL Id: cea-01485765 https://hal-cea.archives-ouvertes.fr/cea-01485765}

Submitted on 9 Mar 2017

HAL is a multi-disciplinary open access archive for the deposit and dissemination of scientific research documents, whether they are published or not. The documents may come from teaching and research institutions in France or abroad, or from public or private research centers.
L'archive ouverte pluridisciplinaire HAL, est destinée au dépôt et à la diffusion de documents scientifiques de niveau recherche, publiés ou non, émanant des établissements d'enseignement et de recherche français ou étrangers, des laboratoires publics ou privés. 


\title{
Ultrathin junctions based on the $\mathrm{LaSrMnO}_{3} / \mathrm{Nb}: \mathrm{SrTiO}_{3}$ functional oxide interface
}

\author{
G. Kurij ${ }^{\text {a,* }}$, L.E. Calvet ${ }^{\text {a }}$, R. Guerrero ${ }^{\text {a }}$, T. Maroutian ${ }^{\text {a }}$, G. Agnus ${ }^{\mathrm{a}}$, A. Solignac ${ }^{\mathrm{b}}$, Ph. Lecoeur $^{\text {a }}$ \\ a Institut d'Electronique Fondamentale, Univ. Paris-Sud, CNRS UMR 8622, 91405 Orsay, France \\ ${ }^{\mathrm{b}}$ DSM/IRAMIS/SPEC, CNRS UMR 3680, CEA Saclay, 91191 Gif sur Yvette, France
}

\section{A R T I C L E I N F O}

Article history:

Received 3 August 2015

Received in revised form 28 March 2016

Accepted 30 March 2016

Available online 2 April 2016

\section{Keywords:}

Functional oxide interface

Schottky junction

Metal-semiconductor contact

Lanthanum strontium manganite

Niobium-doped strontium titanate

\begin{abstract}
A B S T R A C T
High-quality all-oxide thin film metal-semiconductor junctions, fabricated of a half-metallic ferromagnet $\mathrm{La}_{0.7} \mathrm{Sr}_{0.3} \mathrm{MnO}_{3}$ and of a niobium doped n-type semiconductor $\mathrm{SrTiO}_{3}\left(\mathrm{SrTi}_{0.8} \mathrm{Nb}_{0.2} \mathrm{O}_{3}\right)$ by pulsed laser deposition, were studied in terms of their electronic transport properties in a wide temperature range. A fabrication process for ultrathin film metal-semiconductor junctions with micrometre-sized mesas has been established. The current-voltage characteristics of the junctions were found at variance with the conventional thermionic emission theory often applied to explain the transport properties of Schottky contacts. This discrepancy is tentatively ascribed to the very high electric field in the ultrathin $\mathrm{SrTi}_{0.8} \mathrm{Nb}_{0.2} \mathrm{O}_{3}$ layer and its effect on carrier depletion and dielectric constant.
\end{abstract}

(c) 2016 Elsevier B.V. All rights reserved.

\section{Introduction}

Metal-semiconductor interfaces are an integral part of our life: most of the modern electronic devices contain metal-semiconductor contacts [1]. In recent years, Schottky contacts formed by functional oxides $\mathrm{LaSrMnO}_{3}$ (LSMO) and niobium-doped $\mathrm{SrTiO}_{3}$ (Nb:STO) have attracted increasing interest [2-5]. These two perovskites are well known for their remarkable physical properties, widely used in oxide heterostructures and have a high potential for future spintronic applications [6-11].

Nevertheless, in spite of extensive research, the complex interplay of various parameters at oxide metal-semiconductor interfaces remains an open question. In particular, published studies on LSMO/Nb:STO Schottky contacts are usually carried out on Nb:STO bulk-based samples. Only few studies on such junctions in thin film configuration are known, despite their importance for applications in micro- and nanoelectronics. However, in these works relatively thick films were used [12], or the junction structure differs noticeably from the one that we investigated $[13,14]$.

Here we report on fabrication and temperature dependent transport studies of epitaxially grown ultrathin LSMO/Nb:STO metal-semiconductor junctions with micrometre-sized mesas. We show, that for thin film Schottky junctions, transport characteristics near room temperature and at low applied bias can be explained by the thermionic

\footnotetext{
* Corresponding author at: Institut d'Electronique Fondamentale (IEF), Bâtiment 220, Rue André Ampère, 91405 Orsay Cedex, France

E-mail address: georg.kurij@u-psud.fr (G. Kurij).
}

emission theory. For low temperatures and large electric fields, a deviation from thermionic emission theory is observed. We discuss this result regarding the probable effect of electric field- and temperaturedependent permittivity on transport characteristics in ultrathin metalsemiconductor junctions.

The ability to controlled creation of tailored metal-semiconductor interfaces with desired parameters is crucial for the development of novel spintronic devices.

\section{Experimental details}

Bilayers consisting of $\mathrm{SrTi}_{0.8} \mathrm{Nb}_{0.2} \mathrm{O}_{3}$ on top of $\mathrm{La}_{0.7} \mathrm{Sr}_{0.3} \mathrm{MnO}_{3}$ were in situ grown by pulsed laser deposition on $\mathrm{TiO}_{2}$ single terminated, (100) oriented $\mathrm{SrTiO}_{3}$ substrates with $0.1^{\circ}$ miscut. A KrF excimer laser with $248 \mathrm{~nm}$ wavelength and $2 \mathrm{~Hz}$ repetition rate was used. The deposition rates were 0.05 and 0.09 monolayer per pulse for LSMO and Nb:STO respectively. We held the substrate temperature constant at $600{ }^{\circ} \mathrm{C}$ and the oxygen background pressure at $16 \mathrm{~Pa}$ for LSMO and 1.6 Pa for $\mathrm{Nb}$ :STO during the deposition. After the deposition the bilayers were in situ annealed in pure oxygen atmosphere under $1 \mathrm{kPa}$.

In order to analyse surface morphology and crystallographic properties of our samples we performed atomic force microscopy (AFM) measurements, using Veeco Innova model in the contact mode, and X-ray diffraction (XRD) analysis, applying a PANalytical X'Pert Pro diffractometer (Cu-K $\mathrm{K}_{\alpha}$ with $\lambda=1.5406 \AA$ ) in the parallel beam configuration. Layer thicknesses were obtained using X-ray reflectivity technique. We varied the Nb:STO thickness in our samples from $2.4 \mathrm{~nm}$ to $18 \mathrm{~nm}$, and LSMO layer thickness from $50 \mathrm{~nm}$ to $65 \mathrm{~nm}$. 
a)

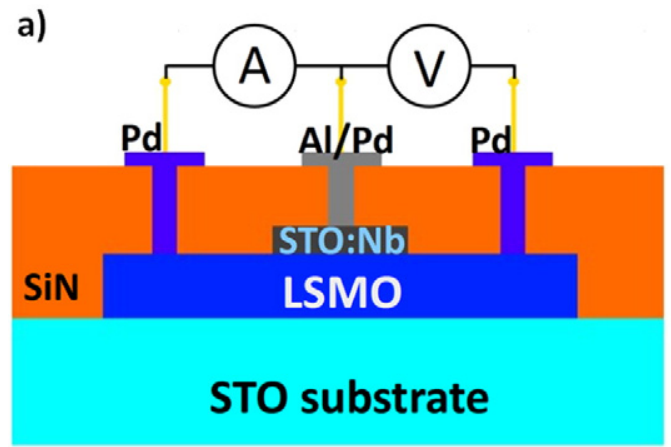

b) $\quad \mathrm{Al} / \mathrm{Pd}$



Fig. 1. a) Schematic side view of a LSMO/Nb:STO junction; b) top view of a junction with connections.

Junctions (Fig. 1a) with different sized mesa areas (from $25 \mu \mathrm{m}^{2}$ to $10^{4} \mu \mathrm{m}^{2}$ ) were fabricated using optical lithography and chemically assisted ion beam etching controlled by a secondary ion mass spectrometer. Two types of junctions were fabricated: type $A P$ with aluminium (Al) and palladium (Pd) pads on top of $\mathrm{Nb}$ :STO and LSMO respectively, to insure good ohmic contacts $[15,16]$, thus forming an asymmetric metal-semiconductor (LSMO/Nb:STO) junction; type $P$ with $\mathrm{Pd}$ on both electrodes, Nb:STO and LSMO, to create an additional non-ohmic contact on the semiconductor side, resulting in a symmetric metalsemiconductor-metal (LSMO/Nb:STO/Pd) junction. In both cases sputtering method was used to form the contacting pads. To connect the junctions to the cryostat for transport measurements we used wire bonding.

Transport measurements were performed in the $300 \mathrm{~K}-77 \mathrm{~K}$ temperature range. Samples were mounted on a vacuum insert and then placed in liquid nitrogen. We measured current-voltage characteristics of our junctions with a Keithley 2636 A source meter. Forward bias direction was defined as the positive voltage applied on the LSMO side (Fig. 1).

In the presented study we focus on the $4 \mathrm{~nm} \mathrm{Nb:STO/50} \mathrm{nm} \mathrm{LSMO}$ sample, with Al connection pads on the $\mathrm{Nb}$ :STO side.

\section{Results}

\subsection{Structural characterization of thin films}

Deposited thin film bilayers demonstrate under the AFM a smooth surface, with root mean square roughness of $0.4 \mathrm{~nm}$. (Fig. 2a). In Fig. 2b X-ray 2-Theta-Omega scans are presented.

Since the intensity of the Nb:STO (002) XRD peak for the samples with ultrathin (2.4-4 nm) Nb:STO layer was not high enough to obtain $\mathrm{Nb}:$ STO lattice parameter, we used as reference the sample with $18 \mathrm{~nm}$ $\mathrm{Nb}$ :STO on top of $50 \mathrm{~nm}$ LSMO. With this method Nb:STO lattice parameter of $0.398 \mathrm{~nm}$ was measured, which is in good agreement with previously reported results for the same level of Nb doping in STO [17].

We conclude from the results of surface and structural analysis that the obtained thin films are of good crystalline quality.

\subsection{Temperature-dependent transport measurements}

$\mathrm{Nb}$ :STO/LSMO junctions of AP type exhibit rectifying behaviour in the whole measured temperature range ( $300 \mathrm{~K}-77 \mathrm{~K}$ ). The rectification ratio was determined as the ratio of current density at $2 \mathrm{~V}$ to that at
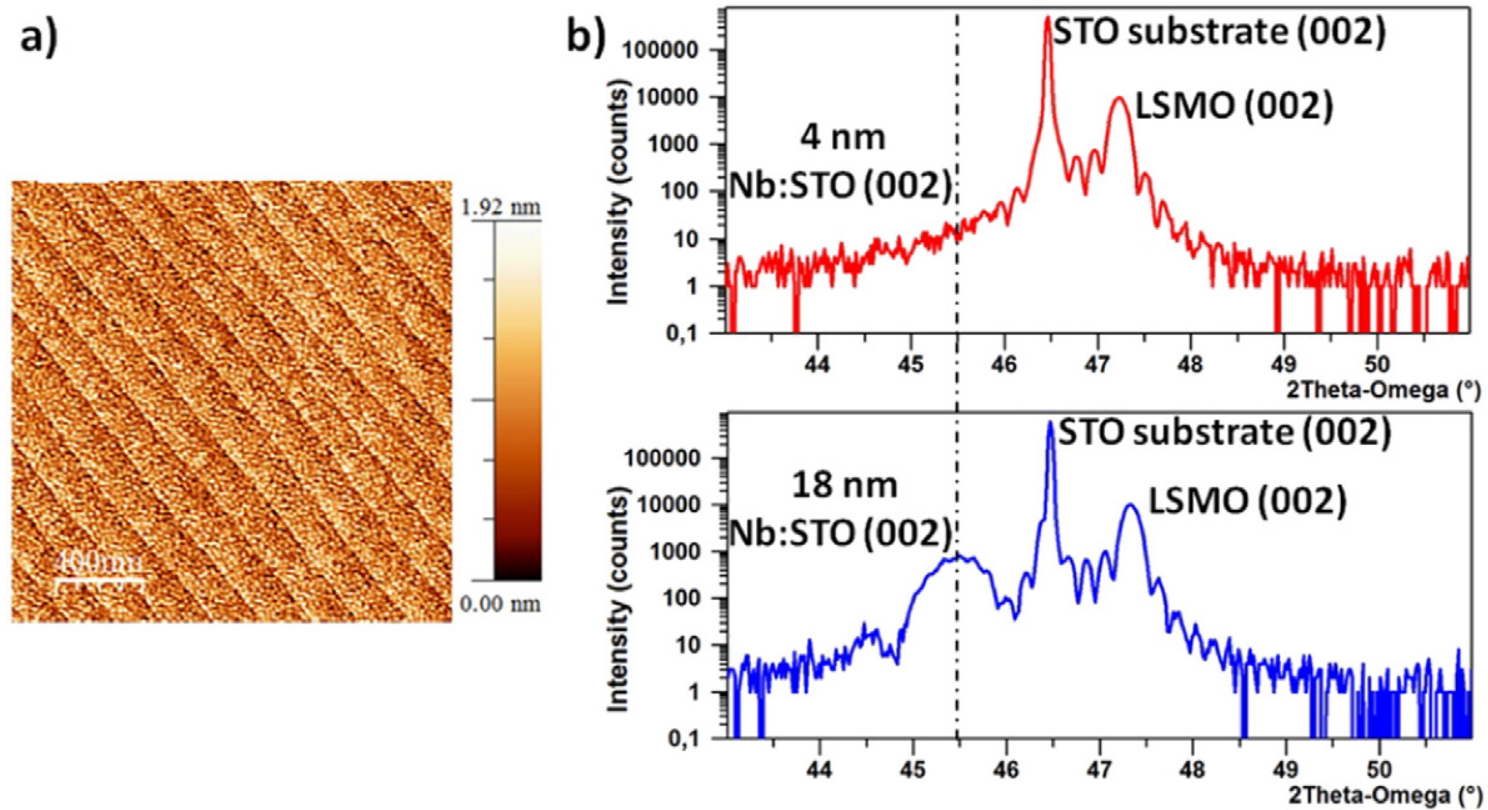

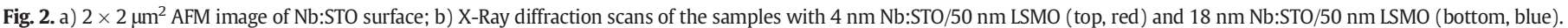


$-2 \mathrm{~V}$. The rectification ratio of the sample with $4 \mathrm{~nm} \mathrm{Nb}$ :STO layer (sample $I$ ) was approximately 30 at low temperature. We explain this relatively low rectification by the ultrathin film structure of the sample, leading to a potential profile in the $\mathrm{Nb}: \mathrm{STO}$ film dominated by both interfaces, $\mathrm{LSMO} / \mathrm{Nb}: \mathrm{STO}$ and $\mathrm{Nb}: \mathrm{STO} / \mathrm{Al}$. In contrast, the rectification ratio of the sample with $18 \mathrm{~nm} \mathrm{Nb:STO} \mathrm{(sample} \mathrm{II)} \mathrm{is} \mathrm{more} \mathrm{than} \mathrm{an}$ order of magnitude higher, indicating a potential profile closer to that of a classical rectifying junction. Another distinction between the two $A P$ samples is a lower current in sample II, especially in the reverse direction. At low temperature the reverse current is at least three orders of magnitude smaller than in sample I and below nano-ampere range, reaching the limit of our measuring system. We observed a faster decrease of the reverse current with decreasing temperature in sample II, compared with sample I. This explains the higher rectification ratio in sample II, especially at low temperature.

The current-voltage characteristics of AP type samples demonstrate a strong temperature dependence (Fig. 3a). In contrast, the sample of $P$ type exhibits symmetric, almost temperature-independent currentvoltage characteristics over the whole measured temperature range (Fig. 3b). This temperature independence indicates a tunnelling mechanism for the current injection through the Nb:STO layer with Pd contacts. On the other hand, we analysed the current-voltage characteristics of AP type junctions using the standard Schottky equation for thermionic emission in the case of a reverse biased junction [18]:

$J=A^{*} T^{2} \exp \left[\frac{-q}{k T}\left(\Phi_{B}-\sqrt{\frac{q E}{4 \pi \varepsilon_{0} \varepsilon_{0 p}}}\right)\right]$,

where $J$ is the current density, $A^{*}$ is the Richardson constant, here $156 \mathrm{~A} \mathrm{~cm}^{-2} \mathrm{~K}^{-2}$ [19], $T$ is the temperature, $q$ is the electron charge, $k$ is the Boltzmann constant, $\Phi_{B}$ is the potential barrier height, $E$ is the electric field at the interface, $\varepsilon_{0}$ is the vacuum permittivity and $\varepsilon_{O p}$ is the optical permittivity. Assuming a homogeneous and temperature independent electric field in the Nb:STO layer, taken as $E=V / d$, with $d$ - the layer thickness, we obtain the following expression:

$J=A^{*} T^{2} \exp \left[\frac{-q}{k T}\left(\Phi_{B}-\sqrt{\frac{q V}{4 \pi d \varepsilon_{0} \varepsilon_{0 p}}}\right)\right]$.

In Eq. (2) the apparent potential barrier $\Phi_{A p p}$ is defined as:

$\Phi_{\text {App }}=\Phi_{B}-\sqrt{\frac{q V}{4 \pi d \varepsilon_{0} \varepsilon_{0 p}}}$.

The graphical representation $\ln \left(J / A^{*} T^{2}\right)$ versus $1 / T$ (Fig. $4 a$ ) should give the apparent potential barrier as the slope of the straight lines obtained for each voltage. By plotting of the apparent potential barrier versus square root of voltage, we can estimate the Schottky barrier height
$\Phi_{B}$ : we find a value of $0.14 \mathrm{eV}$, which is much lower than $0.7 \mathrm{eV}$ predicted by the Schottky-Mott rule or values reported by other authors [20$22]$. The calculated optical permittivity $\varepsilon_{O p}$ of 33 is non-physically large. An explanation for the failure of this conventional analysis of the temperature dependence is thus probably the influence of the high electric field in the semiconductor. Since the thickness of Nb:STO layer in our junctions is extremely low, this results in a high electric field at the interface even for low applied voltage, and in a fully depleted semiconducting layer.

To verify our conclusion we used an alternative form of the Schottky representation: the voltage dependent representation. Starting from the same equation for thermionic emission in a reverse biased contact (Eq. (1)), we plotted $\operatorname{Ln}\left(J / A^{*} T^{2}\right)$ versus square root of voltage (Fig. $4 \mathrm{~b}$ ).

From Fig. 4b we see, that for room temperature the linear fit and thus the $V^{1 / 2}$ dependence of the reverse bias current are very good. But for low temperatures, there is a noticeable deviation from the $V^{1 / 2}$ behaviour for high voltages. Inset in Fig. 4b shows the current-voltage characteristics of sample II. There is a clear difference in forward bias, compared with the sample I (see Fig. 3a). Sample II exhibit in forward bias a transport behaviour as expected for a Schottky junction.

\section{Discussion}

Conduction mechanism in Schottky junctions at low temperature is often explained by tunnelling, with current-voltage characteristics similar to the ones measured for the $P$ type junction (Fig. 3b). But, since in our junctions of $A P$ type the current-voltage characteristics are temperature dependent, tunnelling ansatz does not fit our data correctly.

Some authors, e.g. [23] consider LSMO/Nb:STO junctions as pnjunctions. But the square root of voltage dependent current, as observed in our junctions in a wide temperature range, speaks strongly against the pn-model. Another important indication contradicting the pnmodel, is the drastic drop of current in the AP sample II.

However, an electric field dependent permittivity in Nb:STO was already reported in [24-26] with the following phenomenological equation:

$\varepsilon_{r}(E, T)=\frac{b(T)}{\sqrt{a(T)+E^{2}}}$,

where $a(T)$ and $b(T)$ are temperature dependent parameters. A deviation of capacitance-voltage $(C V)$ characteristics in Nb:STO Schottky junctions from expected linear behaviour was explained in Ref. [25] using Eq. (4). We measured CV characteristics of our devices and also observed a non-linear behaviour. With $\varepsilon(E, T)$ relationship as in Eq. (4) we deduce, that at high electric field the static permittivity in Nb:STO is noticeably lowered. We roughly estimated the depletion width $W_{D}$ in Nb:STO using the equation expressed by S. M. Sze [18], assuming
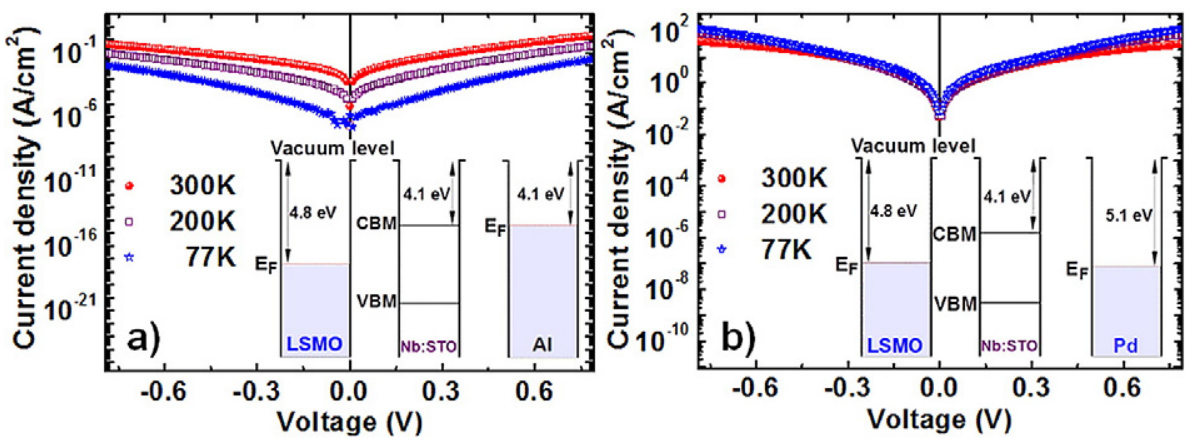

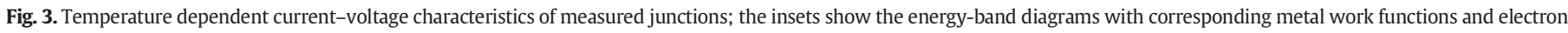

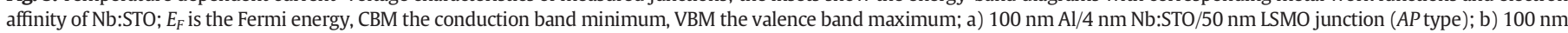
$\mathrm{Pd} / 3 \mathrm{~nm}$ Nb:STO/65 nm LSMO junction ( $P$ type). 

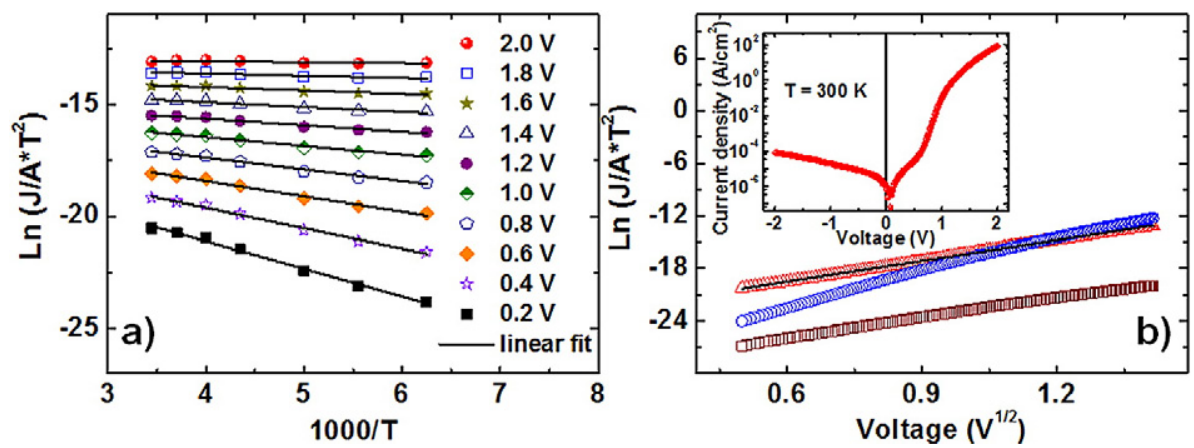

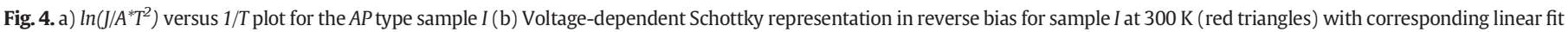
(black line) and $80 \mathrm{~K}$ (blue circles), and for the sample II at $300 \mathrm{~K}$ (brown squares); the inset shows current-voltage characteristic of the sample II at $300 \mathrm{~K}$

the build-in potential $\varphi_{b i}=0.7 \mathrm{~V}$, carrier density $N_{D}=10^{20} \mathrm{~cm}^{-3}$, voltage $V=0$, temperature $T=300 \mathrm{~K}$, and the field dependent permittivity, as calculated in [25], i.e. $\varepsilon_{r}=40$ and $\varepsilon_{r}=180$ for the sample with $4 \mathrm{~nm}$ $\mathrm{Nb}: \mathrm{STO}$ and the reference sample respectively. The carrier density was estimated from capacitance-voltage measurements performed at $300 \mathrm{~K}$. The obtained value is lower than expected for this doping level $\left(\sim 10^{21} \mathrm{~cm}^{-3}\right)$ and an indication for partially inactive dopants. The calculated depletion width $W_{D}$ was found to be close to $5.5 \mathrm{~nm}$ for the junction with $4 \mathrm{~nm} \mathrm{Nb}$ :STO layer and circa $12 \mathrm{~nm}$ for the reference sample with $18 \mathrm{~nm} \mathrm{Nb:STO}$. Thus, we assume that the Nb:STO layer is fully depleted in both types of ultrathin film junctions: $P$ and $A P$.

Assuming a fully depleted Nb:STO layer, the electric field in the positively charged (ionized Nb donors) layer would depend on this static permittivity, thus becoming both: voltage- and temperaturedependent, at variance with the simple $E=V / d$ picture. This effect is a possible explanation for the observed deviation from thermionic emission model at low temperature and large electric field.

\section{Conclusions}

Summarized, we have fabricated and analysed ultrathin all-oxide Schottky junctions formed by the metallic oxide LSMO and heavily doped semiconductor Nb:STO. Transport characteristics at high temperature were analysed in the frame of the thermionic emission theory. At low temperature we observed a possible strong dependence of permittivity on electric field. The result of our analysis points toward an importance of further investigations on complex oxide metal-semiconductor interfaces for spintronic applications.

\section{References}

[1] R.T. Tung, Recent advances in Schottky barrier concepts, Mater. Sci. Eng. R 35 (2001) $1-138$.

[2] M. Minohara, I. Ohkubo, H. Kumigashira, M. Oshima, Band diagrams of spin tunnelling junctions $\mathrm{La}_{0 .} \mathrm{Sr}_{0.4} \mathrm{MnO}_{3} / \mathrm{Nb}: \mathrm{SrTiO}_{3}$ and $\mathrm{SrRuO}_{3} / \mathrm{Nb}: \mathrm{SrTiO}_{3}$ determined by in situ photoemission spectroscopy, Appl. Phys. Lett. 90 (2007) 132123.

[3] A. Sawa, A. Yamamoto, H. Yamada, T. Fujii, M. Kawasaki, J. Matsuno, Y. Tokura, Fermi level shift in $\mathrm{La}_{1}{ }_{\mathrm{x}} \mathrm{Sr}_{\mathrm{x}} \mathrm{MnO}_{3}(\mathrm{M}=\mathrm{Mn}, \mathrm{Fe}, \mathrm{Co}$, and $\mathrm{Ni}$ ) probed by Schottky-like heteroepitaxial junctions with $\mathrm{SrTi}_{0.99} \mathrm{Nb}_{0.01} \mathrm{O}_{3}$, Appl. Phys. Lett. 90 (2007) 252102.

[4] M. Minohara, R. Yasuhara, H. Kumigashira, M. Oshima, Termination layer dependence of Schottky barrier height for $\mathrm{La}_{0.6} \mathrm{Sr}_{0.4} \mathrm{MnO}_{3} / \mathrm{Nb}: \mathrm{SrTiO}_{3}$ heterojunctions, Phys. Rev. B 81 (2010) 235322.

[5] K.G. Rana, T. Yajima, S. Parui, A.F. Kemper, T.P. Devereaux, Y. Hikita, H.Y. Hwang, T. Banerjee, Hot electron transport in a strongly correlated transition-metal oxide, Sci. Rep. 3 (2013) 1274.
[6] J.-H. Park, E. Vescovo, H.-J. Kim, C. Kwon, R. Ramesh, T. Venkatesan, Direct evidence for a half-metallic ferromagnet, Nature 392 (1998) 794-796.

[7] T. Yajima, Y. Hikita, H.Y. Hwang, A heteroepitaxial perovskite metal-base transistor, Nat. Mater. 10 (2011) 198-201.

[8] S.A. Wolf, D.D. Awschalom, R.A. Buhrman, J.M. Daughton, S. von Molnar, M.L. Roukes, A.Y. Chtchelkanova, D.M. Treger, Spintronics: a spin-based electronics: vision for the future, Science 294 (2001) 1488-1495.

[9] A.M. Haghiri-Gosnet, T. Arnal, R. Soulimane, M. Koubaa, J.P. Renard, Spintronics: perspectives for the half-metallic oxides, Phys. Status Solidi 201 (2004) 1392-1397.

[10] M. Bibes, A. Barthelemy, Oxide spintronics, IEEE Trans. Electron Devices 54 (2007) 1003.

[11] W. Han, X. Jiang, A. Kajdos, S.-H. Yang, S. Stemmer, S.S.P. Parkin, Spin injection and detection in lanthanum- and niobium-doped $\mathrm{SrTiO}_{3}$ using the Hanle technique, Nat. Commun. 4 (2013) 2134

[12] H.B. Lu, S.Y. Dai, Z.H. Chen, Y.L. Zhou, B.L. Cheng, K.J. Jin, L.F. Liu, G.Z. Yang, X.L. Ma, High sensitivity of positive magnetoresistance in low magnetic field in perovskite oxide p-n junctions, Appl. Phys. Lett. 86 (2005) 032502

[13] K. Ueda, K. Tozawa, H. Asano, Inversion of magnetoresistance in $\mathrm{La}_{1}-{ }_{\mathrm{x}} \mathrm{Sr}_{\mathrm{x}} \mathrm{MnO}_{3} / \mathrm{Nb}-$ doped $\mathrm{SrTiO}_{3} / \mathrm{CoFe}$ junctions, J. Korean Phys. Soc. 63 (2013) 706-710.

[14] H.C. Yu, M.P. Wang, Y.L. Jia, C. Chen, W.C. Yang, C.D. Xia, L.F. Liu, Y.G. Wang, Transmission electron microscopy study of multilayer $\mathrm{p}-\mathrm{n}$ hetero-junction $\mathrm{La}_{0.9} \mathrm{Sr}_{0.1} \mathrm{MnO}_{3} / \mathrm{SrNb}_{0.05} \mathrm{Ti}_{0.95} \mathrm{O}_{3}$ thin films, Thin Solid Films 519 (2011) 2079-2082.

[15] L. Mieville, D. Worledge, T.H. Geballe, R. Contreras, K. Char, Transport across conducting ferromagnetic oxide/metal interfaces, Appl. Phys. Lett. 73 (1998) $1736-1738$.

[16] H.B. Michaelson, The work function of the elements and its periodicity, J. Appl. Phys. 48 (1977) 4729-4733.

[17] T. Tomio, H. Miki, H. Tabata, T. Kawai, S. Kawai, Control of electrical conductivity in laser deposited $\mathrm{SrTiO}_{3}$ thin films with $\mathrm{Nb}$ doping, J. Appl. Phys. 76 (1994) 5886-5890.

[18] S.M. Sze, Physics of Semiconductor Devices, third ed. Wiley, New Jersey, 2007.

[19] T. Shimizu, N. Gotoh, N. Shinozaki, H. Okushi, The properties of Schottky junctions on Nb-doped $\mathrm{SrTiO}_{3}$ (001), Appl. Surf. Sci. 117 (118) (1997) 400-405.

[20] M. Minohara, Y. Furukawa, R. Yasuhara, H. Kumigashira, M. Oshima, Orientation dependence of the Schottky barrier height for $\mathrm{La}_{0.6} \mathrm{Sr}_{0.6} \mathrm{MnO}_{3} / \mathrm{SrTiO}_{3}$ heterojunctions, Appl. Phys. Lett. 94 (2009) 242106.

[21] A. Ruotolo, C.Y. Lam, W.F. Cheng, K.H. Wong, C.W. Leung, High-quality all-oxide Schottky junctions fabricated on heavily doped $\mathrm{Nb}: \mathrm{SrTiO}_{3}$ substrates, Phys. Rev. B 76 (2007) 075122.

[22] F.M. Postma, R. Ramaneti, T. Banerjee, H. Gokcan, E. Haq, D.H.A. Blank, R. Jansen, J.C. Lodder, Epitaxial diodes of a half-metallic ferromagnet on an oxide semiconductor, J. Appl. Phys. 95 (2004) 7324-7326.

[23] P. Han, K.-J. Jin, H.-B. Lu, Q.-L. Zhou, Y.-L. Zhou, G.-Z. Yang, The mechanism study on transport properties in perovskite oxide p-n junctions, Appl. Phys. Lett. 91 (2007) 182102.

[24] R.A. van der Berg, P.W.M. Blom, J.F.M. Cillessen, R.M. Wolf, Field dependent permittivity in metal-semiconducting $\mathrm{SrTiO}_{3}$ Schottky diodes, Appl. Phys. Lett. 66 (1995) 697-699.

[25] S. Suzuki, T. Yamamoto, H. Suzuki, K. Kawaguchi, K. Takahashi, Y. Yoshisato, Fabrication and characterization of $\mathrm{Ba}_{1}-{ }_{\mathrm{x}} \mathrm{K}_{\mathrm{x}} \mathrm{BiO}_{3} / \mathrm{Nb}$-doped $\mathrm{SrTiO}_{3}$ all-oxide-type Schottky junctions, J. Appl. Phys. 81 (1997) 6830-6836.

[26] T. Yamamoto, S. Suzuki, K. Kawaguchi, K. Takahashi, Temperature dependence of the ideality factor of $\mathrm{Ba}_{1}-{ }_{\mathrm{x}} \mathrm{K}_{\mathrm{x}} \mathrm{BiO}_{3} / \mathrm{Nb}$-doped $\mathrm{SrTiO}_{3}$ all-oxide-type Schottky junctions, Jpn. J. Appl. Phys. 37 (1998) 4737-4746. 